\title{
The novel coronavirus (SARS-CoV-2) infections in China: prevention, control and challenges
}

\author{
Sheng Zhang ${ }^{1}$, Meng Yuan Diao², Liwei Duan³, Zhaofen Lin ${ }^{3^{*}}$ and Dechang Chen ${ }^{1 *}$ (1)
}

(C) 2020 Springer-Verlag GmbH Germany, part of Springer Nature

\section{Dear Editor,}

Since December 2019, an outbreak of novel coronavirus (SARS-CoV-2) that began from Wuhan, Hubei Province, has rapidly spread to 34 provincial-level divisions of China [1] and 25 countries around the world [2]. Up to February 15, 2020, 68,586 cases in China and 526 cases in other countries have been identified as having COVID19 (eFigure 1). The estimated mortality rate is $3.1 \%$ in Wuhan, $2.8 \%$ in Hubei Province, $0.6 \%$ in other provinces of China, $2.4 \%$ in China and $0.4 \%$ in other countries, respectively.

Faced with such a grim situation, the Chinese government has taken a series of unprecedented rigorous measures. First, all the 31 provincial-level divisions in China mainland have launched the highest level of responding mechanism for major public health emergency. Second, Wuhan and other 12 cities in Hubei Province have consecutively shut down all outbound transportation channels and suspended public transportation. Third, the State Council announced that both the Spring Festival holiday and winter vacation will be extended. Fourth, two emergency makeshift hospitals (Huoshenshan and Leishenshan hospitals) with a total capacity of 2600 beds were built and more than ten cabin hospitals were renovated with more than 10,000 beds in Wuhan. Fifth, more

\footnotetext{
*Correspondence: linzhaofen@hotmail.com; chendechangsh@hotmail. com

${ }^{1}$ Department of Critical Care Medicine, Ruijin Hospital, Shanghai Jiao Tong University School of Medicine, Shanghai 200025, China

${ }^{3}$ Department of Emergency and Critical Care Medicine, Changzheng Hospital, Naval Medical University (Second Military Medical University), Shanghai 200433, China

Full author information is available at the end of the article

Sheng Zhang, Meng Yuan Diao and Liwei Duan have contributed equally to this work.
}

than 30,000 members from military and public hospitals have successively headed out to Wuhan and other cities in Hubei Province.

Although the number of confirmed cases is still increasing, the increasing rate has showed a downward trend from February 4, 2020 (eFigure 2). Despite Hubei Province, Guangdong, Henan, Zhejiang, Hunan and Anhui are the top five provinces with respect to the ranking order of confirmed cases. The increasing rates of confirmed cases in these provinces also showed a downward trend recently (eFigure 3). Using a real-time Bayesian estimation model and a reported serial interval of COVID-19 [3, 4], we computed time-dependent reproduction number, $R(t)$, which is defined as the expected number of secondary cases that one primary case will generate during infectious disease transmission. As shown in Fig. 1, $R(t)$ has showed a downward trend in Wuhan, Hubei Province, outside Hubei, and China from January 27, 2020, to February 10, 2020. The downward trend of $R(t)$ indicates that the prevention and control measures may be effective, although long-term effects remain to be evaluated.

However, we still confront a number of great challenges. First, source of SARS-CoV-2 remains pendent and the population is generally susceptible to the new virus. Second, although human-to-human spread [3] is thought to occur mainly via respiratory droplets and close contact, fecal-oral transmission or vertical transmission may also be a means of transmission. Third, asymptomatic cases with COVID-19 have been reported [5], and several places in China have reported confirmed cases without clear transmission chain, which indicates that there may be some infected cases still wandering among population and spreading virus. Fourth, as the Spring Festival

\section{实




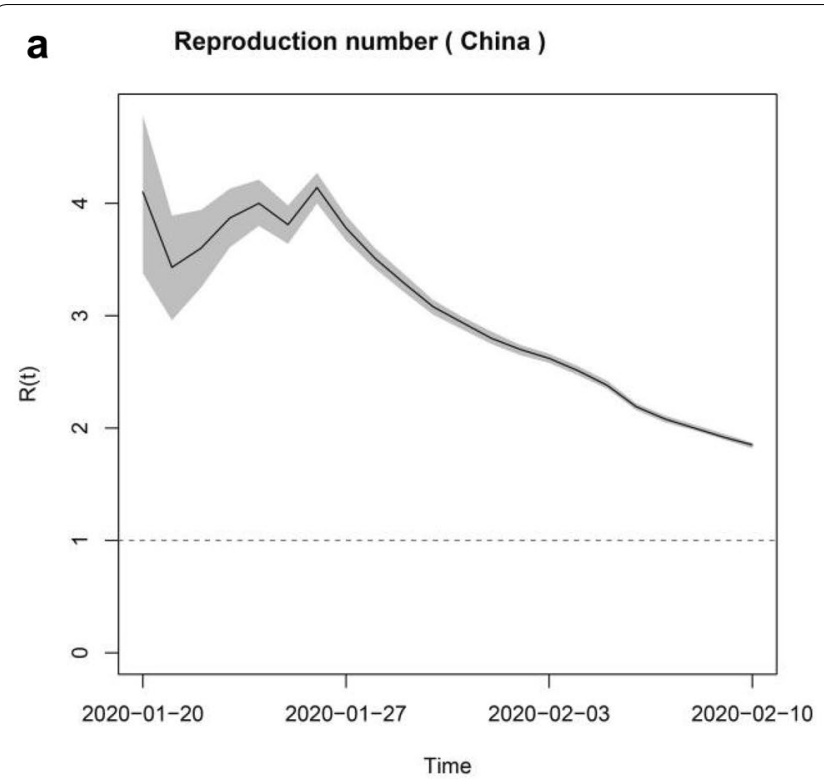

b Reproduction number ( Outside Hubei)

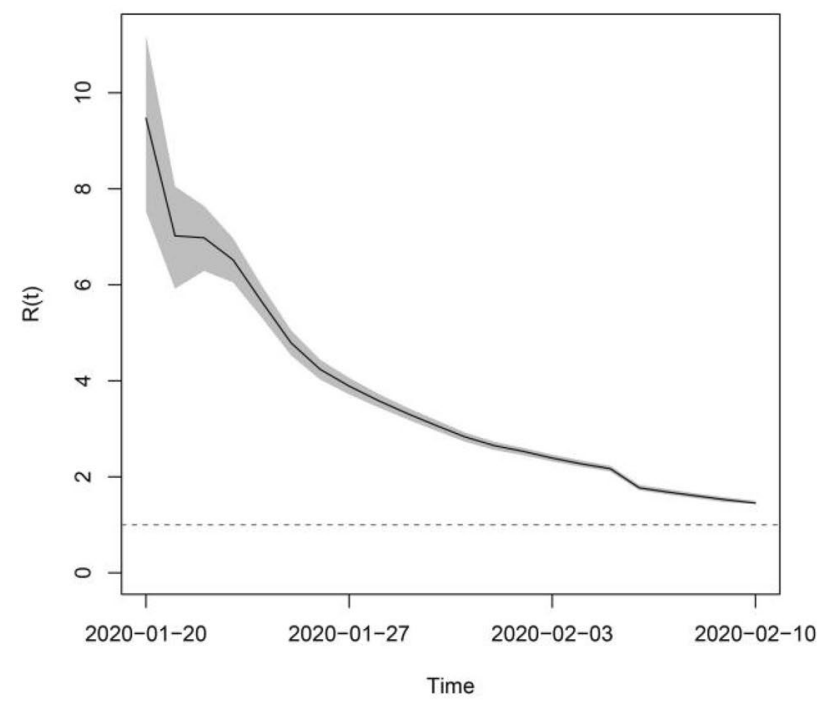

C

Reproduction number ( Hubei )
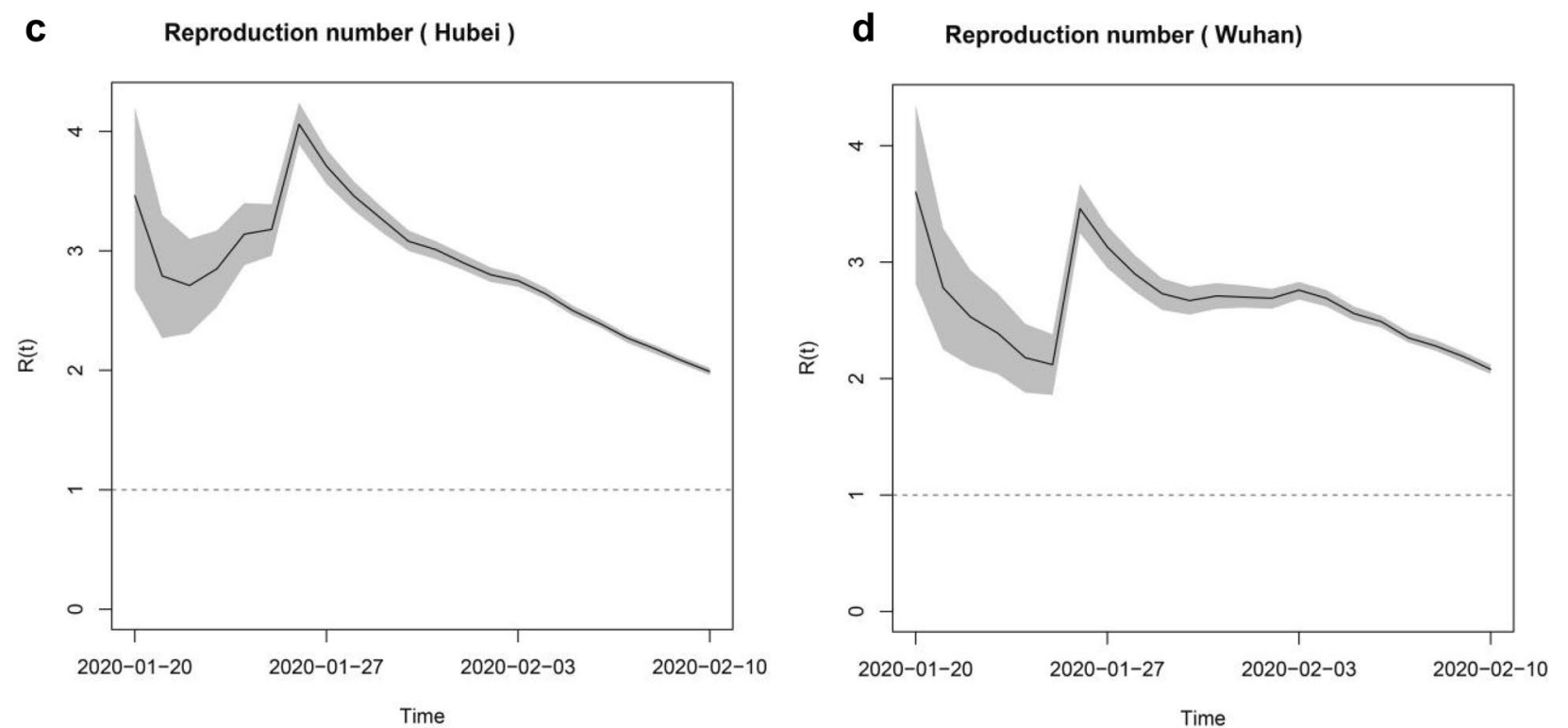

Fig. 1 Real-time reproduction number of COVID-19 in China, outside Hubei, Hubei and Wuhan. Real-time reproduction number $R(t)$ with $95 \%$ confidence interval of COVID-19 in a China, b outside Hubei, $\mathbf{c}$ Hubei Province and d Wuhan. The estimates of $R(t)$ were truncated at February 10, 2020, because the diagnosis criteria for COVID-19 are adjusted since February 12, giving a sharp increase in number of new cases who was identified as having COVID-19. Incorporating these data for $R(t)$ derivation would obtain misleading results

holiday nears its end, hundreds of millions people will migrate from hometown to metropolitan areas for work. Such a large scale of population migration proposes a huge challenge for epidemic control and prevention.

\section{Electronic supplementary material}

The online version of this article (https://doi.org/10.1007/s00134-020-05977-9) contains supplementary material, which is available to authorized users.

\section{Author details}

${ }^{1}$ Department of Critical Care Medicine, Ruijin Hospital, Shanghai Jiao Tong University School of Medicine, Shanghai 200025, China. ${ }^{2}$ Department of Critical Care Medicine, Affiliated Hangzhou First People's Hospital, Zhejiang University School of Medicine, Hangzhou 310006, Zhejiang, China. ${ }^{3}$ Department of Emergency and Critical Care Medicine, Changzheng Hospital, Naval Medical University (Second Military Medical University), Shanghai 200433, China.

\section{Authors' contribution}

SZ and MYD helped in the conception and design of the study. SZ and LWD contributed to data collection and analysis. MYD helped in the first draft writing. ZFL and CDC contributed to the final approval of the version to be 
submitted. All authors commented on previous versions of the manuscript and read and approved the final manuscript.

\section{Compliance with ethical standards}

\section{Conflicts of interest}

The authors declared no conflict of interest.

\section{Publisher's Note}

Springer Nature remains neutral with regard to jurisdictional claims in published maps and institutional affiliations.

Accepted: 19 February 2020

Published online: 2 March 2020

\section{References}

1. National health commission of the People's Republic of China (2020) The latest situation of new coronavirus pneumonia. https:/www.nhc.gov.cn/ xcs/yqfkdt/202002/4a1b1ec6c03548099de1c3aa935d04fd.shtml
2. World Health Organization (2020) Coronavirus disease (COVID-19) Situation Report-26. https://www.who.int/docs/default-source/coronaviruse/ situation-reports/20200215-sitrep-26-covid-19.pdf?sfvrsn=a4cc6787_2

3. Li Q, Guan XH, Wu P et al (2020) Early transmission dynamics in Wuhan, China, of novel coronavirus-infected pneumonia. N Engl J Med. https:// doi.org/10.1056/NEJMoa2001316

4. Bettencourt LM, Ribeiro RM (2008) Real time Bayesian estimation of the epidemic potential of emerging infectious diseases. PLoS ONE 3(5):e2185. https://doi.org/10.1371/journal.pone.0002185

5. Rothe C, Schunk M, Sothmann P et al (2020) Transmission of 2019-nCoV infection from an asymptomatic contact in Germany [published online January 28, 2020]. N Engl J Med. https://doi.org/10.1056/NEJMc2001272 